\title{
Pharmacy-Drug Development and Technology Novel Drug Delivery
}

\section{Dhanapal*}

Department of Pharmacognosy and Phytochemistry, Sanjo College of Pharmaceutical Studies, Vellapara, Palakkad, India

*Corresponding Author: Dhanapal, Department of Pharmacognosy and Phytochemistry, Sanjo College of Pharmaceutical Studies, Vellapara, Palakkad, India.

Received: July 17, 2019; Published: July 25, 2019

DOI: $10.31080 /$ ASPS.2019.03.0356

Drug delivery system favors a good therapeutic effect by proper administration of drugs to the patients. In order to get a desired and safe therapeutic effect this novel drug delivery system will have its focus on the different technologies, formulations and the method for transporting the drug in our body. The method of drug delivery is of at most important as it alters the efficacy of the drug. This subject of novel drug delivery system aligns with its allied fields like molecular biology, polymer science and pharmaceutics. This system is concerned about the physical mechanisms like osmosis, dissolution, diffusion and biochemical mechanisms like nano particles, liposomes, monoclonal antibodies, gene therapy etc. NDDS mainly purposed to combine with the specific target region, thereby increases the therapeutic efficacy and in turn reduces the side or toxic effects. NDDS designs the formulation in such a way that the drug is loaded in any of the natural or synthetic polymers. The carriers can be made slowly degradable, stimuli-reactive, and even targeted (e.g., by conjugating them with specific antibodies against certain characteristic components of the area of interest).
In the present scenario of research, the novel drug delivery system is concerned to get rid of harmful side effect, to avoid drug deterioration and loss and for accumulation in the specific site of need, and to increase the bioavailability of drug. If we consider the present decade, researchers are extensively and intensively working on this subject. Thus because of advancement in NDDS the maximum efficacy of the drug is expected with the minimum wastage of drug.

\section{Volume 3 Issue 8 August 2019 \\ (c) All rights are reserved by Dhanapal.}

